\title{
A database of dynamics
}

The Dynameomics database houses nativestate and high-temperature unfolding simulation data for the $\mathbf{1 0 0}$ most populated protein folds; such information provides insights into protein function beyond structure alone.

As undoubtedly useful as three-dimensional reconstructions of protein structures are, they do not tell the whole story of protein function. A structure is a static snapshot of the most stable conformation, but in reality, proteins are in constant motion, even when they are not doing anything particularly interesting. This wiggling and jiggling is crucial for protein function. It can also help explain why mutated proteins cause disease, and it has even been suggested to be responsible for protein evolvability, but experimentally studying protein dynamics is still quite challenging.

Computer simulations can help fill in some of the gaps. With advances in computational power, improved algorithms and reduced costs, molecular dynamics simulations have become potent tools to investigate protein dynamics. "[Such] simulations allow one to 'see' the various forms of a protein constituting the native ensemble, including structures that fluctuate sometimes dramatically about the average," explains Valerie Daggett of the University of Washington. "It is often these transient excursions that are critical to function."

Daggett has been applying simulations on a large scale to try to obtain new insights into protein function. Over the last ten years, in the context of a project called 'Dynameomics', her team has been building up a database of molecular dynamics simulations of the native states and high-temperature unfolding pathways for a large number of proteins. In their newest release, they now present simulation data for 100 representative protein folds, collected by performing 11,000 individual molecular dynamics simulations on more than 2,000 proteins from 218 different organisms. The database of simulations is publicly available at http://www.dynameomics.org/.

Daggett's group has applied a careful strategy to select diverse protein folds to analyze. Using three existing protein fold classification systems - named SCOP, CATH and Dali- they identified domains from protein structures available in the Protein Data Bank and clustered them into what they call 'metafolds'. They aim to simulate at least one member from each protein metafold. They run molecular dynamics simulations at $298 \mathrm{~K}$ for at least 31 nanoseconds to sample native state dynamic fluctuations, as well as hightemperature (498K) unfolding simulations. Although experimental dynamic information is not available for most of their targets, notes Daggett, "the simulations compare quite well with experiments where experimental data are available."

The Dynameomics database is useful both for answering questions about specific proteins as well as for obtaining general insights into protein folding and function. For example, the simulations can map conformational changes with atomic-level detail, which can then be tested by experiments, Daggett notes. "We already have several examples of dynamic behavior linked to function that was discovered through the molecular dynamics simulations and not at all evident in the static average experimental structures," she says. She also believes that the collection of simulations will be useful for obtaining fundamental insights into the rules of proteins folding, a question that remains a central challenge in the protein biochemistry field.

Daggett's group is also performing simulations of single-nucleotide polymorphismcontaining mutant human proteins implicated in disease. For some of these proteins, the simulations provided insights into why distal mutations caused structural disruptions at the active site. The database also contains simulations for several proteins from thermophilic organisms. Analyzing the native state and unfolding dynamics of these proteins as compared to their mesophilic equivalents may help address the question of what makes these proteins stable at high temperatures - a question that structural analysis alone has not yet been able to fully answer.

With combined efforts and technical advances in both theoretical simulations and experimental methods for studying protein dynamics, scientists will gain insights into protein folding and function, the development of disease and perhaps even devise new therapeutic solutions.

\section{Allison Doerr}

\section{RESEARCH PAPERS}

van der Kamp, M.W. et al. Dynameomics: a comprehensive database of protein dynamics. Structure 18, 423-435 (2010). 